\title{
The Effect of Long-distance Jump Training on the Athletes' Performance in the Late Course of Sprint in Track and Field
}

\author{
Leipo Chen
}

South China Institute of Software Engineering, Guangzhou University Guangzhou 510000, China.

Keywords: Track and field sprint, the Training of long distance jump, Effect analysis.

\begin{abstract}
The track and field sprint, which is an anaerobic energy supply project, is a typical project of speed competition. Its primary energy material ATP - CP which will run out in 4 to 5 seconds, can only maintain the energy of the athletes for 30-40 meters. How to improve the late course performance of the sprinters is the key factor to get a better result. Based on the teaching practice of many years, the author adopts a new training method to improve the performance of athletes in the late course of the track and field sprinting. The method mainly adopts long distance jump training, supplemented by short and medium distance jump training, and combines the traditional running high-speed endurance level training. The author sets up the experimental group, which a long jump training is given priority to, and control group, which adopts traditional high-speed endurance level training. By comparing the experimental results of two groups of athletes, the author analyzes the sprint mechanism and the energy supply and saving system to explore how does the training of long distance jump affect the athletes’ performance in the late course of sprint in track and field.
\end{abstract}

\section{Introduction}

Sprint project is a high intensity exercise that human body sustained high speed run under anoxic conditions. It occupies very important position in the modern Olympic history and always been the focus of widespread. In the first world modern Olympic Games, the best results for the 100 and 400 meters were 12.0 and 54.2 seconds respectively. By the second Olympic Games, the 100-meter mark had risen to 10.8 seconds. At the 2008 Beijing Olympics, Jamaican athlete Usain Bolt set a new world record with 9.69 seconds. While the sprinting level of the world has been greatly improved, the sprinting project in China has been in a state of stagnation for many reasons. The two main reasons are the difference of human races and the method of training. Human race cannot be changed. However, the improvement and innovation of training methods are quite promising.

\section{Study Subjects and Methods}

\subsection{Subjects.}

We choose our three grade sprinting teams in our college, grade 15, 16 and 17 respectively.

\subsection{Energy Supply Mechanism Analysis and Data Method.}

The researches of Atp-cp system, anaerobic glycolysis system and energy conservation have taken a great step now. But the sprinting project in China has been in a state of stagnation for many reasons. The two main reasons are the difference of human races and the method of training. Race cannot be changed. However, the improvement and innovation of training methods are quite promising.

\subsection{The Method of Grouping and Segmented Statistical Experimental Comparison.}

We set up an experimental group and a control group, each of them contains 10 people. To assess the training effect objectively, we decided to record the data of two groups before and after the training. The original data before the training about the first 60 meters, the latter 40 meters, and 100 meters are recorded in table 1 , table 2 . The data after training are recorded in table 3 , table 4 . 
Table 1. The original average score of grade 15, 16 and 17 in the experimental group.

\begin{tabular}{|c|c|c|c|c|}
\hline Grade & Population & First 60m & Latter $40 \mathrm{~m}$ & $100 \mathrm{~m}$ \\
\hline 15 & 10 & $7.62 \mathrm{~s}$ & $5.10 \mathrm{~s}$ & $12.72 \mathrm{~s}$ \\
\hline 16 & 10 & $7.60 \mathrm{~s}$ & $5.13 \mathrm{~s}$ & $12.73 \mathrm{~s}$ \\
\hline 17 & 10 & $7.59 \mathrm{~s}$ & $5.11 \mathrm{~s}$ & $12.70 \mathrm{~s}$ \\
\hline
\end{tabular}

Table 2. As the original average scores of grades 15,16 and 17 of the control group.

\begin{tabular}{|c|c|c|c|c|}
\hline Grade & Population & First 60m & Latter $40 \mathrm{~m}$ & $100 \mathrm{~m}$ \\
\hline 15 & 10 & $7.60 \mathrm{~s}$ & $5.05 \mathrm{~s}$ & $12.65 \mathrm{~s}$ \\
\hline 16 & 10 & $7.50 \mathrm{~s}$ & $5.10 \mathrm{~s}$ & $12.60 \mathrm{~s}$ \\
\hline 17 & 10 & $7.58 \mathrm{~s}$ & $5.00 \mathrm{~s}$ & $12.58 \mathrm{~s}$ \\
\hline
\end{tabular}

Table 3. The average score of 100 meters after training in the experimental group of grades 15, 16 and 17.

\begin{tabular}{|c|c|c|c|c|}
\hline Grade & Population & First $60 \mathrm{~m}$ & Latter $40 \mathrm{~m}$ & $100 \mathrm{~m}$ \\
\hline 15 & 10 & $7.10 \mathrm{~s}$ & $4.30 \mathrm{~s}$ & $11.40 \mathrm{~s}$ \\
\hline 16 & 10 & $7.07 \mathrm{~s}$ & $4.32 \mathrm{~s}$ & $11.39 \mathrm{~s}$ \\
\hline 17 & 10 & $7.08 \mathrm{~s}$ & $4.33 \mathrm{~s}$ & $11.41 \mathrm{~s}$ \\
\hline
\end{tabular}

Table 4. The average score of 100 meters after the training in control group of grades 15, 16 and 17.

\begin{tabular}{|c|c|c|c|c|}
\hline Grade & Population & First $60 \mathrm{~m}$ & Latter $40 \mathrm{~m}$ & $100 \mathrm{~m}$ \\
\hline 15 & 10 & $7.11 \mathrm{~s}$ & $4.73 \mathrm{~s}$ & $11.84 \mathrm{~s}$ \\
\hline 16 & 10 & $7.08 \mathrm{~s}$ & $4.81 \mathrm{~s}$ & $11.89 \mathrm{~s}$ \\
\hline 17 & 10 & $7.09 \mathrm{~s}$ & $4.82 \mathrm{~s}$ & $11.91 \mathrm{~s}$ \\
\hline
\end{tabular}

\section{Long-Distance Jumps}

There are two kinds of jumps in track and field training. One is jump in place, such as standing vertical jump and Switch Split leap, and the other is displacement jump, such as standing jump, multi-stage jump, multi-stage frog jump, single hop, etc. Due to the different in the displacement jump distance, it can be divided into a short jump, mid-range jump and long jump, short jump is that a kind of displacement jump that the jump distance below 100 meters, 100-150 m for mid-range jump, more than 150 meters for the long-distance jump. The author's training method for the experimental group is to take the long-distance jump as the main subject, take the terminal distance jump as the auxiliary subject, and take a proper arrangement of the round trip run as high-speed endurance level training. In the control group, the author adopted the traditional training method of short distance jumping based on high-speed endurance level, and each group was arranged 1-2 times per week. After 1 year of training, the experimental group was compared with the control group.

We can see form table1-4, there's not much difference in first 60 meters, but the difference in later 40 meters is significant. Compare with the original data the experimental group decreased by more than 0.7 seconds, while control group decreased only slightly more than 0.3 seconds. After training, the experimental group improved $0.4 \mathrm{~s}$ more than the control group in the later 40 meters. This data shows that the long jump training can improve the performance of sprinter in $100 \mathrm{~m}$ sprint greatly. According to biomechanics, back step running and stride leap is a movement progress that knee and hip stretching with rapid contraction of muscles. This movement progress can intense stimulate and strengthen the muscle group that running act could not. So, by repeating a long jump training can improve muscle contraction force and the high-speed endurance level.

\section{The Late Course of Sprint in Track and Field}

Studies have shown that, in 100-meters sprint, sprinters often begin to slow down during 50 to 70 meters, and the maximum speed can only be maintained a short distance, about 14 percent to 16 percent. Therefore, sprint performance is affected or restricted by the high-speed endurance level. For sprinters, high-speed endurance level is the ability to maintain the fastest speed to the finish line. 
In 200 meters sprint project, almost no domestic sprinters can reach the finishing line with a time cost that equal to the twice of the average of their best score in 100 meters sprint. But most of the foreign excellent athletes can reach or exceed theirs. such as the former Soviet Union Bauer Zoff, 100 meters,10s,200 meters, 20s; Monia from Italy,100 meters, 10.10s, 200 meters,19.7s. It indicates that there is a significant gap in high-speed endurance level between sprinters of China and foreign sprinters. Therefore, it is the goal of Chinese athletes to improve the high-speed endurance level.

\section{Energy Supply and Energy Saving}

Sprinting is an extreme sports program. Its main features are rapid muscle contraction, rapid movement of the body and rapid displacement. Sprinters need to reach the body fastest frequency or the fastest displacement. This requires the energy supply system to produce the maximum energy in a short time, so its metabolism is characterized by a high degree of hypoxia in motion. Energy is supplied mainly by phosphoric acid system and glycogen. The research of exercise physiology has confirmed that phosphocreatine (CP) and triphosphate (ATP) are stored in muscle cells, which release a large amount of energy when decomposed, providing energy for rapid muscle contractions. Are to be highlighted, just as muscle fiber cross-sectional area and strength increase under the stimulus of effective training enlargement, CP - ATP system and anaerobic glycolysis system will get compensatory to strengthen or improve accordingly under the stimulation of long-term and effective training. The "capacity" of the energy reserve in the (sarcoplasmic muscle cells) is also "amplified". This fact told us that the extreme long-distance jump (increase jump distance and shorten skip time) is more exciting for the stimulation of the energy supply system than the high-speed endurance training of running. So, it has a better affect to the sprinter's performance in late course of sprint. It is the same result of our experiment before. On the other hand, ineffective training stimulus, will not improve "two systems". "Capacity” also won't increase. for example, the intensity of small and medium-sized stimulus, someone did the experiment: They provide pure creatine to the long-term strength of small and medium-sized training athletes, found that almost all the pure creatine wasted in physiological metabolism excretion, supplement creatine is valid only on those athletes who take long-term intensive training. Sports medicine animal experiments also prove that: when ATP decomposition and release energy, CP will discompose and release energy for ATP synthesis, the process still need other support materials or auxiliary materials, such as vitamins $\mathrm{E}$ and $\mathrm{C}$, In particular, vitamin $\mathrm{C}$, when vitamin $\mathrm{C}$ deficiency, the activity of ATP in the animal heart and skeletal muscles decreased significantly, and the activity of ATP was restored when the normal amount of vitamin C was supplied. Therefore, vitamin C can enhance the activity of ATP and promote the synthesis and utilization of ATP.

Although effective intensive training can improve the CP - ATP system, strengthen the function of anaerobic glycolysis system, and amplify the energy capacity of sarcoplasm - muscle cells. For sprint, a muscle contraction extreme sport, is still a drop in the bucket. The maximum power of APT can only be maintained for 6 seconds. According to this calculation, it can only support for the first 60 meters in a 100 meters sprint. The energy for first 60 meters is mainly supplied by the storage of APT.The LA system start while the sprinter begins to slow down in the late course. After that, the ATP begin to synthesis and supply the energy we needed. This process is also related with energy supplement and energy saving. According to the study, excessive muscle tension can affect blood circulation, which can also aggravate the "viscosity" of the muscle fibers and hinder the "channel" of the energy supply system. In addition, excessive muscle tension can result in increased muscle resistance, muscle stiffness, reduced productivity, and increased energy consumption. The better Muscle relaxation ability is, the better ATP resynthesis efficiency will be. In this sense, given in the case of constant energy, energy conservation is particularly important. Coordinating relaxation create artificial prerequisites for saving energy, and energy conservation provide the condition to improve performance of sprinter in the late course and to supply high-speed energy. 


\section{Conclusion}

\subsection{Experiment Conclusion.}

The training method that take the long-distance jump as the main subject, take the terminal distance jump as the auxiliary subject and take the round trip run as high-speed endurance level training is very effective to improve the sprinters performance.

\subsection{Conclusion.}

Effective training can thicken the cross-cutting area of muscle fibers and strengthen the cp-atp system and anaerobic glycolysis system.

\subsection{Conclusion.}

The coordination and relaxation technique of sprinting is an important artificial condition for energy conservation, and it is also an effective way to shorten the distance of deceleration and improve the performance of late course sprinting.

\section{The Analysis of Related Factors that Influence the Performance of Sprinters and the Method to Improve it.}

\subsection{Related Factors that Influence the Performance of Sprinters.}

\subsubsection{Pace and Stride Length.}

Sprinting is an extreme sports program that human body have to keep high-speed moving in anaerobic conditions. In the whole process of sprinting, stride length and stride frequency play a decisive role in running speed. athletes must keep a good combination of both and manage to raise both or one of them. In comparison, the improvement of stride length is more important to improve sprint performance.

\subsubsection{External States and Internal Causes.}

This paper analyzes the factors that influence sprint speed from external state and internal causes and constructs the velocity genesis model to analyze and demonstrates the relationship between two aspects. The relationship between them is that the external factors should reach the corresponding indexes when we are developing the intrinsic elements. Base on this theory we have propose the optimum index range and velocity distance curve.

\subsubsection{Speed Power.}

Stride length of the World best sprinter is significantly higher than ordinary players, and no significant differences on step length, the main reason is that frequency of leg swinging of the best sprinter is higher than ordinary sprinters.

\subsubsection{Factor that Influence the Overall Rhythm.}

The frequency of the step is more important when accelerating, because we need to get rid of the static state quickly. When we reach the top speed, which is the high-speed maintenance phase, the stride length is more important, because we need to minimize muscle tension and maintain the high speed to the end. Thus, stride length is the dominant factor in the competition.

\subsubsection{Speed Barrier.}

The "speed barrier" is due to the fact that the physical quality and technical movement of the sprinter lag behind further need of speed improvement. The amount of exercise, intensity and training method has no new stimulation to the body.

\subsection{The Method to Improve the Performance of Sprinters.}

\subsubsection{Improve the Starting Speed, Strengthen the Running Ability in the Middle, and Pay} Attention to the Finishing Line Technology.

Sprinters' starting and running effects have a great impact on performance, and the starting effect is influenced by physical quality, nervous system and psychology. In order to improve the starting speed of sprinters, we should pay more attention on the starting part in sprinting training. We should arrange the sprinting training in time that our body is energetic, pay attention to the importance of the "preparation" movement and improve technology of pushing off the blocks and ability of acceleration. 
The middle part of sprinting is an important stage. To improve the performance in this part, we have to develop the strength of the adductor group, which is the muscle group we use when we swagging our leg. Finish technique is the last kind spring skill, the specific requirements of the Finish technology is that: when the athletes get to the position that one step distance away from the finish line, their upper body rapidly move forward, ran across the finish line with their chest or shoulder hitting toward the upside of finish line, and then gradually slow down the pace.

\subsubsection{Develop the Ability of Step Frequency and Step Length Harmoniously.}

The development trend of sprint technology is that the training should pay more attention to the standardization of sprint technology. The structure of sprinting technology tends to rationalize, we can tell this form the greater emphasis on swing technology. The development of step frequency and length is synchronized; It increases the distance that the sprinter keep their high speed and better performance in the late course; Greater emphasis on coordination and relaxation.

\subsubsection{Strengthen the Rear Pedal and Swing Arm Technical Training, Improve the Post-Kick Effect and Swing Arm Technique.}

The swing arm technique is composed of the elbow Angle of the swing arm, the direction of the swing arm and the amplitude of the swing arm. The function of the swing arm is to increase the post-kick force, maintain the balance of the body, and improve the running speed.

\subsubsection{Strengthen the Speed Strength Training and Improve the Speed Ability.}

The acceleration capability and the high-speed endurance level are two important factors to determine the sprint performance.

Special strength training methods must have the same structure, the same muscle working process, the same energy supply process as the sport competition.

The special maximum speed capability refers to the max speed the sprinter can get and the duration of the max speed. In the training of sprinters, the extension of the hip joint should be emphasized. In the strength training we should pay more attention to the lower limbs. The contraction speed of the flexor muscle of the knee joint should be improved at the same time.

\section{References}

[1]. Wang linen. A brief analysis of the methods about the training and recovery of sprinters [J]. Wisdom, 2015, (10): 297-298.

[2]. Xiao hangman. A brief analysis of the methods about the training and recovery of sprinters in athletics [J]. Sports science 2016, (05): 49-51.

[3]. Fang youngling. Classification of modern sports track and field events and requirements for various athletes [J]. Beijing sports society, 2015, (05): 102-338.

[4]. Li Xingjian. The difference between long-distance running and sprinter in track and field events [J]. China sports press, 2015, (09): 509-611.

[5]. Fan mingy an. Training and training of sprinters in track and field in China [J]. People education press, 2016, (08): 3382-5503.

[6]. Liang fanny. Different training and requirements corresponding to different sports programs [M]. People education press, 2015, (08): 411-600.

[7]. Lug xiaoyi. Factors affecting sprint speed and solutions [J]. China sports press, 2017, (07): 445-668. 